\title{
Viraje tuberculínico en pacientes con artritis inflamatorias crónicas y terapia biológica
}

\author{
Osvaldo Luis Cerda' ${ }^{1}$, María de los Angeles Correa', Amelia Granel², Ana Marcos², Claudia Giraldo³, Oscar Rillo², \\ Emilce Edith Schneeberger, Gustavo Citera ${ }^{1}$ \\ ${ }^{1}$ Instituto de Rehabilitación Psicofísica, Buenos Aires, Argentina. ${ }^{2}$ Hospital San Roque de Gonnet, La Plata, Argentina. ${ }^{3}$ Hospital General de \\ Agudos Enrique Tornú, Buenos Aires Argentina.
}

\section{Resumen}

Introducción: El bloqueo de los mediadores inflamatorios producido por las terapias biológicas se asocia a un aumento de las infecciones oportunistas, como infección por Mycobacterium tuberculosis (MT), por lo que se recomienda realizar intradermorreacción de derivado proteico purificado (PPD) antes de iniciar dicho tratamiento. Teniendo en cuenta la situación endémica de tuberculosis (TBC) en nuestro país y la inmunosupresión/anergia de los pacientes con artritis inflamatorias crónicas, nos planteamos si es necesario monitorear la infección de MT luego de iniciado el tratamiento biológico.

Objetivos: Evaluar la frecuencia de viraje de PPD y la asociación del viraje con infección activa por MT u otras variables.

Materiales y métodos: Se incluyeron pacientes con Artritis Reumatoidea (AR), Artritis Idiopática Juvenil (AIJ) y Espondiloartritis (EsP) en tratamiento con agentes Anti-TNF $\alpha$, Tocilizumab $\mathrm{y} / 0$ Abatacept. Los pacientes debían tener una PPD negativa $(<5$ $\mathrm{mm}$ ) basal y una segunda PPD realizada entre los 2 y 22 meses posteriores. Se consignaron datos sociodemográficos (edad, género, estatus social, hacinamiento), antecedentes patológicos (desnutrición, alcoholismo, tuberculosis y sus contactos), tipo de enfermedad reumática y tiempo de evolución, índices de actividad (RAPID-3, BASDAI), de función (BASFI, HAQ-II) y tratamiento concomitante con corticoides (CT), drogas modificadoras de la enfermedad (DME) y terapia biológica (TB). Se consideró viraje de PPD a una variación $\geq$ a $5 \mathrm{~mm}$ entre dos pruebas realizadas con intervalo entre 2 meses y 2 años. En caso de viraje se recomendó seguimiento por Neumonología e Infectología. Análisis estadístico: las variables categóricas se compararon por $\mathrm{Chi}^{2}$ y test exacto de Fisher y las continuas por T-test o Mann-Whitney. Regresión logística múltiple.

Resultados: Se incluyeron 85 pacientes, $78,8 \%$ eran mujeres, con una escolaridad mediana de 12 años. El 98,8\% tenía residencia urbana, $12,9 \%$ tenía criterios de hacinamiento y $8,2 \%$ ingresos inferiores a la línea de pobreza. 5,9\% de los pacientes había padecido TBC con cumplimiento de tratamiento completo y

\section{Abstract}

Introduction: The blockade of inflammatory mediators produced by biological therapies is associated with an increase of opportunistic infections such as Mycobacterium tuberculosis (MT), so it is recommended to perform the intradermal purified protein derivative test (PPD) before starting such treatment. Given the endemic situation of tuberculosis (TB) in our country and immunosuppression/ anergy of patients with chronic inflammatory arthritis, we wonder whether if it's necessary to monitor the infection of MT after starting biological treatment.

Objectives: To evaluate the frequency PPD seroconversion and its association with active TB infection and other disease variables.

Materials and methods: Patients with Rheumatoid Arthritis (RA), Juvenile Idiopathic Arthritis (JIA) and Spondyloarthritis (Spa) receiving treatment with anti-TNF, Tocilizumab and/or Abatacept agents were included. Patients had to have one basal PPD negative $(<5 \mathrm{~mm})$ and a second one was performed between 2 and 22 months later. We collected data regarding: socio-demographics (age, gender, social status, overcrowding), medical history (malnutrition, alcoholism, previous tuberculosis and contacts), type of rheumatic disease and disease duration. Disease activity was assessed by RAPID-3 and BASDAI, functional capacity by BASFI and $H A Q-I I$. Other treatments were also consigned. PPD conversion was considered as a variation $\geq 5 \mathrm{~mm}$ between two PPD performed with interval between 2 months to 2 years. Statistical analysis: Categorical variables were compared by $\mathrm{Chi}^{2}$ and Fisher exact test, continuous by T-test or Mann-Whitney. Multiple logistic regression. Results: 85 patients were included, $78.8 \%$ were women, with a median schooling of 12 years, $98.8 \%$ had urban residence, and $8.2 \%$ were under the lower income poverty line. $5.9 \%$ of patients had suffered TBC with full treatment compliance and $2.4 \%$ reported previous TBC contact. $74.1 \%$ of patients had RA, $16.5 \%$ Psoriatic arthritis (PsA) and 4.7\% AlJ and Ankylosing Spondylitis (AS). With regard to treatment, $18.8 \%$ received more than $10 \mathrm{mg}$ daily of oral steroids, $91.8 \%$ DMARDs (84.7\% Methotrexate and $21.2 \%$

\section{Correspondencia}

Gustavo Citera, Jefe de la Sección Reumatología, Instituto de Rehabilitación Psicofísica. gustavocitera@gmail.com 
2,4\% reportó contactos con TBC. En cuanto al tipo de artropatía inflamatoria crónica, el $74,1 \%$ de los pacientes tenían $A R$, el $16,5 \%$ Artritis Psoriásica (APs) y 4,7\% tanto AlJ como Espondilitis Anquilosante. Con respecto al tratamiento, 18,8\% recibía más de 10 mg diarios de esteroides orales, el 91,8\% DME, (84,7\% Metotrexato y $21,2 \%$ Leflunomida). El 75,3\% recibía terapia Anti-TNF $\alpha(31,8 \%$ Etanercept, 21,2\% Adalimumab, 17,6\% Infliximab, 3,5\% Golimumab y $1,2 \%$ Certolizumab), el $15,3 \%$ Tocilizumab y el $9,4 \%$ Abatacept. De los 85 pacientes, 8 viraron $(9,4 \%)$. El viraje fue más frecuente en varones $62,5 \%$ vs mujeres $37,5 \%(p=0,009)$ y en aquellos con mayor tiempo de evolución de enfermedad en meses [X226 \pm 109 vs $\mathrm{X} 130 \pm 105(p=0,017)]$. Esta asociación se mantuvo luego de ajustar para otras variables. Todos los pacientes que presentaron viraje recibieron isoniacida (INH) profiláctica y sólo un paciente, con factores de riesgo, desarrolló tuberculosis activa.

Conclusión: La frecuencia del viraje tuberculínico en pacientes con artritis inflamatorias crónicas fue baja y se asoció a sexo masculino y mayor tiempo de evolución de la enfermedad.

Palabras clave: test de tuberculina, artritis, biológicos.
Leflunomide). $75.3 \%$ received anti-TNF therapy (31.8\% Etanercept, $21.2 \%$ Adalimumab, $17.6 \%$ Infliximab, 3.5\% Golimumab and 1.2\% Certolizumab), $15.3 \%$ Tocilizumab and $9.4 \%$ Abatacept. Eight patients $(9.4 \%)$ developed PPD conversion. The shift was more frequent in men $62.5 \%$ vs women $37.5 \%(\mathrm{p}=0.009)$ and in those with a longer disease duration [X226 \pm 109 vs $X 130 \pm 105(p=0.017)$ ]. This association remained after adjusting for other variables. All patients who developed PPD conversion received prophylactic isoniazid and only one patient with risk factors developed active TB. Conclusion: The frequency of PPD conversion in patients with chronic inflammatory arthritis was low and was associated with male gender and longer disease duration.

Key words: tuberculin test, arthritis, biologicals.

\section{Introducción}

Las artritis inflamatorias crónicas son patologías discapacitantes que requieren un tratamiento adecuado y oportuno. Uno de los tratamientos más efectivos son los agentes biológicos, los cuales tienen un aceptable perfil de seguridad, aunque incrementan el riesgo de infecciones, entre ellas las oportunistas ${ }^{1-2}$. Entre los agentes biológicos, los inhibidores del TNF- $\alpha$ (Tumor Necrosis Factor-Alpha) son los pioneros y por ende los más utilizados. El TNF- $\alpha$ tiene un rol clave en la formación y mantenimiento de los granulomas encargados de contener patógenos intracelulares, como Mycobacterium tuberculosis (MT). En pacientes en tratamiento con antagonistas del TNF- $\alpha$ se ha descripto un incremento de la tasa de infección por MT de hasta 4 veces ${ }^{3-4}$.

Argentina es un país con incidencia mediana de tuberculosis (TBC), en 2011 se notificaron 10.618 casos (tasa de incidencia: 26,6/100.000) al Programa Nacional, 640 personas murieron por TBC durante el 20105. La Prueba de Intradermorreacción de derivado proteínico purificado (PPD) permite evaluar hipersensibilidad mediada por linfocitos $\mathrm{T}$ a proteínas de $\mathrm{MT}^{6}$; sin embargo, esta respuesta puede no ser inmunogénica en pacientes con enfermedades autoinmunes, resultando en un valor negativo7.

La prueba de Mantoux (PPD) es uno de los pocos test desarrollados en siglo XIX que continúa vigente en la medicina actual. Es el único método ampliamente disponible para monitoreo de la TBC. Dada su larga historia, parecería sorprendente que ciertos aspectos en su interpretación generen aún controversias ${ }^{8}$. El valor de corte marcador de infección varía según la epidemiología de la región y tipo de paciente. En nuestro país, el Consenso Argentino de TBC (2009) determina para la población general un valor $\geq 10 \mathrm{~mm}$, $\mathrm{y}$ en caso de pacientes inmunocomprometidos y contactos de alto riesgo un valor $\geq 5 \mathrm{~mm}^{9}$.

La PPD evalúa hipersensibilidad retardada (mediada por linfocitos T) a proteínas de MT. La reacción se produce siempre que haya existido una primera exposición a las proteínas bacilares, sea vacunación (BCG) o infección micobacteriana. Una prueba negativa expresa ausencia de hipersensibilidad y se interpreta en general como ausencia de contacto previo.

Sin embargo pueden suceder dos situaciones:

- Que el individuo pierda con el tiempo la capacidad de respuesta. Esto puede observarse en pacientes de edad avanzada infectados/vacunados en su juventud más allá de 
15 años y no infectados posteriormente ${ }^{10}$.

- Ausencia de reacción, descripto en pacientes con enfermedades autoinmunes con compromiso de la respuesta $\mathrm{Th} 1^{11}$.

Existen otras situaciones no relacionadas a la capacidad de respuesta del paciente, en las cuales la respuesta de la PPD puede modificarse, como diferencias en la administración del derivado y/o modo de lectura o fenómeno de estímulo o "booster". La conversión o viraje representa infección latente o reciente.

Elfenómeno “booster” es unfenómenodehipersensibilidad ante el intenso estímulo antigénico y puede persistir hasta 60 días posteriores al test de $\mathrm{PPD}^{8}$, el cual se produce como consecuencia de realizar la PPD en forma repetida en el intento de detectar infección nueva en contactos recientes, exposición ocupacional y pacientes inmunocomprometidos.

Teniendo en cuenta que Argentina es endémica para TBC, que las terapias biológicas inhiben mediadores inflamatorios encargados del control de la infección y la anergia inmunológica que pueden presentar algunos pacientes, nos proponemos evaluar la frecuencia de viraje de PPD en pacientes con artropatías autoinmunes en tratamiento con Terapia Biológica (TB), analizar la asociación entre viraje de PPD e infección activa por MT y analizar otras variables que pudieran afectar el viraje de la prueba de PPD.

\section{Materiales y métodos}

Estudio multicéntrico, observacional, retrospectivo, de pacientes con artritis inflamatorias crónicas, en el cual participaron tres centros de reumatología: dos de la Ciudad Autónoma de Buenos Aires (Instituto de Rehabilitación Psicofísica y Hospital de Agudos General Enrique Tornú) y uno de la ciudad de La Plata (Hospital San Martín de La Plata). Se incluyeron pacientes de la consulta ambulatoria con diagnóstico de Artritis Reumatoidea (AR), según criterios ACR $1987^{12}$ y ACR/EULAR 2010 ${ }^{13}$, Artritis Idiopática Juvenil (AIJ) según criterios ILAR ${ }^{14}$, Espondiloartritis (EsP) según criterios ASAS para EsP axial ${ }^{15}$ y EsP periférica ${ }^{16}$, Artritis Psoriásica (APs) según criterios CASPAR ${ }^{17}$, que estuvieran en tratamiento con agentes biológicos como antagonistas del TNF- $\alpha$ (Etanercept, Adalimumab, Infliximab, Certolizumab, Golimumab), inhibidor de la interleuquina 6 (IL-6) (Tocilizumab) o de la coestimulación del linfocito T CTLA4 (Abatacept). Todos los pacientes incluidos debían tener una primera prueba de PPD negativa $(\leq 5 \mathrm{~mm})$ previo al inicio de la TB. Se excluyeron pacientes con diagnóstico de TBC activa o latente previa, pacientes que tuvieran dos reacciones de PPD realizadas fuera del tiempo del intervalo establecido y pacientes con enfermedades infecciosas agudas o crónicas, que pudieran interferir con los resultados de la $\mathrm{PPD}^{8}$.

Los pacientes que cumplieron con los criterios de inclusión fueron citados al centro de atención médica correspondiente y firmaron consentimiento informado para participar en el estudio. Se consignaron datos sociodemográficos como edad, sexo, ocupación, tipo de residencia (urbana, rural), y escolaridad. También se investigaron condiciones asociadas a transmisión de MT como:

- Hacinamiento (según OMS): convivencia de 3 o más personas por habitación como dormitorio.

- Bajo peso (según OMS): déficit calórico proteico que se traduce en índice de masa corporal (IMC) $<18,5$.

- Alcoholismo (según OMS): ingesta diaria de alcohol superior a 20 a 40 gramos en la mujer y 40 a 60 gramos en el hombre ${ }^{18}$.

- Pobreza (según Instituto Nacional de Estadística y Censo19): ingreso mensual inferior a $\$ 1500$ argentinos para una familia con 3 hijos (08/2013), siendo esta fecha coincidente con el estudio.

Se recabaron datos de la enfermedad como: tiempo de evolución, presencia de comorbilidades, tratamiento recibido dentro del año previo al inicio de la terapia biológica, considerando esteroides a altas dosis como prednisona o su equivalente en dosis de $\geq 10 \mathrm{mg} /$ día o $\geq 3$ aplicaciones de esteroides parenterales al año, uso de DME (Drogas Modificadoras de la Enfermedad) y tipo de agente biológico administrado y dosis. Todos los datos se obtuvieron a través de la entrevista con el paciente y mediante la revisión de historias clínicas.

También se evaluó actividad de enfermedad mediante cuestionario RAPID3 ${ }^{20}$ e índice compuesto DAS28 para pacientes con AR y APs y BASDAI $^{21}$ para pacientes con EsP axial (ax). La capacidad funcional fue evaluada mediante HAQ- ${ }^{22}$ en pacientes con AR y APs y $\mathrm{BASFI}^{14}$ en pacientes con EsPax.

Posteriomente se les solicitó a todos los pacientes participantes del estudio una segunda prueba de PPD, en un intervalo de tiempo posterior a la primera PPD de 2 a 22 meses. Se respetó este tiempo para evitar en el primer caso "booster" y en el segundo la pérdida del estímulo antigénico $^{6-8}$. La Prueba de PPD consistió en la inyección de $0,1 \mathrm{ml}$ de PPD (equivalentes a 2 unidades de tuberculina), con posterior medición de pápula a las 48-72 horas por lectores ciegos entrenados. Se definió viraje como la variación mayor a $5 \mathrm{~mm}$ en el diámetro de la pápula con respecto a la primera pruebas de PPD 9 .

Análisis estadístico: Estadística descriptiva, las variables continuas se expresan en mediana y rango intercuartilo (RIC) y las categóricas en frecuencia y porcentaje. Los datos continuos fueron analizados por T-Test y Mann-Whitney y los categóricos por $\mathrm{Chi}^{2}$ y test exacto de Fisher. Se realizó regresión logística múltiple utilizando la presencia de viraje como variable dependientes con el fin de evaluar las variables asociadas independientemente al mismo. Se consideró significativa una $\mathrm{p}<0,005$.

\section{Resultados}

Se incluyeron 85 pacientes, 63 (74,1\%) con diagnóstico de AR, 14 (16,5\%) APs, 4 (4,7\%) de AIJ y 4 (4,7\%) EsPax. Sesenta y siete pacientes $(78,8 \%)$ eran de sexo femenino. La mayoría de los pacientes $(98,8 \%)$ vivían en zonas urbanas y uno sólo 


\begin{tabular}{|c|c|}
\hline Variable & $\mathrm{n}=85$ \\
\hline Sexo masculino, $\mathbf{n}(\%)$ & $18(21,2)$ \\
\hline \multicolumn{2}{|l|}{ Patologías: } \\
\hline Artritis Reumatoidea, $\mathrm{n}(\%)$ & $63(74,1)$ \\
\hline Artritis Psoriásica, n (\%) & $14(16,5)$ \\
\hline Artritis Idiopática Juvenil & $4(4,7)$ \\
\hline Espondilitis Anquilosante & $4(4,7)$ \\
\hline Pobreza & $7(8,2)$ \\
\hline Hacinamiento, n (\%) & $11(12,9)$ \\
\hline \multicolumn{2}{|l|}{ Ocupación } \\
\hline Amas de casa, n (\%) & $26(30,6)$ \\
\hline Profesionales, n (\%) & $13(15,3)$ \\
\hline Jubilados, n (\%) & $11(12,9)$ \\
\hline Administrativo, n (\%) & $10(11)$ \\
\hline Comerciantes, n (\%) & $8(9,4)$ \\
\hline Obreros de la construcción, n (\%) & $3(3,5)$ \\
\hline Trabajadores de la salud, n (\%) & $2(2,4)$ \\
\hline Estudiantes, $\mathrm{n}(\%)$ & $1(1,2)$ \\
\hline Desocupado, n (\%) & $10(11,8)$ \\
\hline Tipo de factores de riesgo (en 14 pacientes) & $14(16,5)$ \\
\hline Diabéticos tipo 2, n (\%) & $9(10,6)$ \\
\hline Alcoholismo, $\mathrm{n}(\%)$ & $1(1,2)$ \\
\hline TBC con tratamiento completo, $\mathrm{n}(\%)$ & $5(5,9)$ \\
\hline Contacto con portadores de TBC, n (\%) & $2(2,4)$ \\
\hline \multicolumn{2}{|l|}{ Tratamiento DME concomitante } \\
\hline Metotrexato, n (\%) & $72(84,7)$ \\
\hline Leflunomida, $\mathrm{n}(\%)$ & $18(21,2)$ \\
\hline Hidroxicloroquina, n (\%) & $6(7,1)$ \\
\hline Sulfazalasina, $\mathrm{n}(\%)$ & $1(1,2)$ \\
\hline \multicolumn{2}{|l|}{ Tratamiento biológico } \\
\hline Etanercept, n (\%) & $27(31,8)$ \\
\hline Adalimumab, n (\%) & $18(21,2)$ \\
\hline Infliximab, n (\%) & $15(17,6)$ \\
\hline Certolizumab, n (\%) & $1(1,2)$ \\
\hline Golimumab, $\mathrm{n}(\%)$ & $3(3,5)$ \\
\hline Abatacept, n (\%) & $8(9,4)$ \\
\hline Tocilizumab, n (\%) & $13(15,3)$ \\
\hline Corticoterapia, n (\%) & $42(49,4)$ \\
\hline Prednisona >10 mg/día, n (\%) & $16(18,8)$ \\
\hline
\end{tabular}

TBC: Tuberculosis; DME: Drogas Modificadoras de la Enfermedad.

Tabla 1. Características clínicas, sociodemográficas y terapéuticas de los pacientes con enfermedades reumáticas evaluados.

provenía de una zona rural. La edad mediana fue 52 años (RIC 46-60), y el tiempo mediano de evolución fue 11,5 años (RIC 4,8-16). La escolaridad mediana fue 12 años (RIC 7-14). Once pacientes $(12,9 \%)$ presentaban condiciones de hacinamiento y $7(8,2 \%)$ vivían por debajo de la línea de pobreza. Catorce pacientes $(16,5 \%)$ presentaron factores de riesgo para TBC, alguno de ellos más de un factor de riesgo asociado (17 factores de riesgo en 14 pacientes): 9 (10,6\%) diabetes tipo 2, 1 $(1,2 \%)$ alcoholismo, $2(2,4 \%)$ pacientes tuvieron contacto con portadores de TB. $5(5,9 \%)$ presentaron antecedentes de TBC con tratamiento completo. Setenta y ocho pacientes $(91,8 \%)$ recibían DME combinado a la terapia biológica, 42 (49,4\%) corticoides, y 16 de ellos (18,8\%) tenían esteroides en dosis $\geq 10 \mathrm{mg}$ (Tabla 1).

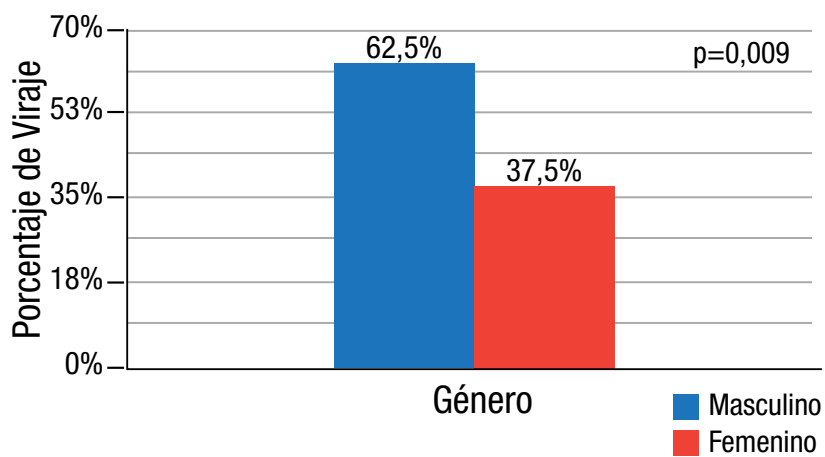

Figura 1. Asociación entre viraje y género.

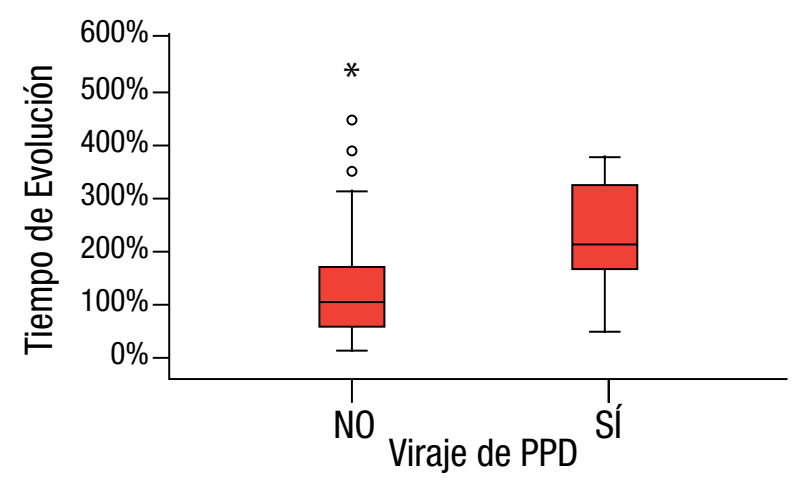

Pacientes que no viraron vs Pacientes que sí viraron, según tiempo de evolución de la enfermedad en meses [X226 \pm 109 vs X130 $\pm 105(p=0,017)]$.

Figura 2. Asociación entre viraje y tiempo de evolución de la enfermedad.

Se observó viraje de PPD en 8 (9,4\%). El mismo fue significativamente más frecuente en varones que en mujeres $62,5 \%$ vs $37,5 \%(p=0,009)$ y en aquellos con mayor tiempo de evolución de la enfermedad $[\mathrm{X} 226 \pm 109$ vs $\mathrm{X} 130 \pm 105$ $(\mathrm{p}=0,017)]$. (Figuras 1 y 2$)$

Estas asociaciones se mantuvieron en el análisis de regresión logística luego de ajustar por edad, sexo y tiempo de evolución de la enfermedad. No encontramos asociación entre viraje de PPD y actividad de la enfermedad (DAS28, RAPID3, BASDAI), discapacidad (HAQ-A, BASFI) o calidad de vida (RAQoL, ASQoL, PSAQoL), así como tampoco con otras variables sociodemográficas o con comorbilidades.

Todos los pacientes que presentaron viraje continuaron seguimiento por Infectología o Neumonología, y recibieron Isoniacida (INH) profiláctica a dosis de $300 \mathrm{mg} /$ día. Luego de un período de seguimiento mediano de 14,8 meses, solo un paciente desarrolló TBC pulmonar activa. Este paciente presentaba como posibles factores de riesgo diabetes tipo 2; antecedentes de TBC tratada y corticoterapia a altas dosis. (Tabla 2)

\section{Discusión}

En el presente estudio, investigamos la frecuencia de viraje tuberculínico en pacientes con enfermedades inflamatorias 


\begin{tabular}{|c|c|c|c|c|c|c|c|c|c|c|c|}
\hline Paciente & AIC & Sexo & Ocupación & Pobreza & Hacinamiento & DBT & Historia de TBC & СтC & DME & Isoniacida & TBC Activa \\
\hline 1 & APs & $M$ & Profesional & NO & NO & NO & NO & NO & NO & sí & NO \\
\hline 2 & $\mathrm{AR}$ & $\mathrm{F}$ & Ama de casa & NO & NO & NO & NO & NO & Sí & Sí & NO \\
\hline 3 & $\mathrm{AR}$ & $\mathrm{F}$ & Desocupado & NO & NO & NO & NO & NO & Sí & Sí & NO \\
\hline 4 & APs & $M$ & Comerciante & NO & Sí & NO & NO & NO & Sí & Sí & NO \\
\hline 5 & AR & $\mathrm{F}$ & Comerciante & NO & NO & NO & N0 & sí & Sí & Sí & NO \\
\hline 6 & AR & $M$ & Comerciante & NO & NO & NO & N0 & NO & NO & Sí & NO \\
\hline 7 & AR & M & Obrero & NO & NO & sí & sí & sí & sí & sí & sí \\
\hline 8 & APs & $M$ & Comerciante & NO & NO & NO & sí & NO & Sí & Sí & NO \\
\hline
\end{tabular}

AIC: Artritis inflamatoria crónica; DBT: Diabetes; TBC: Tuberculosis; CTC: Corticoterapia: Prednisona >10 mg/día; DME: Droga Modificadora de la Enfermedad; AR: Artritis Reumatoidea; APs: Artritis Psoriásica.

Tabla 2. Características de los 8 pacientes que viraron prueba de PPD.

crónicas tratados con terapias biológicas, basándonos en el incremento de riesgo de TB en los pacientes tratados con terapias biológicas ${ }^{17}$. Varios registros europeos han reportado un aumento de la frecuencia de tuberculosis en pacientes con AR y tratamiento biológico ${ }^{4-23}$. La frecuencia fue mayor en aquellos que no realizaron un adecuado testeo para TB latente, en quienes las formas extrapulmonares (principalmente ganglionares) fueron las más frecuentes ${ }^{23}$. En un estudio coreano se analizó el viraje de PPD en pacientes con AR con terapia biológica y se observó que 28 de 86 pacientes tuvieron conversión positiva de la PPD durante el tratamiento, y a raíz de estos resultados sugirieron el monitoreo anual de los pacientes previamente negativos para PPD en tratamiento biológico $^{24}$. En nuestro estudio observamos viraje en el $9,4 \%$ de la población estudiada, y las variables asociadas a la presencia de viraje fueron el sexo masculino y el mayor tiempo de evolución de la enfermedad. Sin embargo, sólo 1 paciente desarrolló TBC activa, aunque es de destacar que todos los pacientes que viraron recibieron profilaxis para TBC latente.

Existen reportes de una mayor frecuencia de anergia a la PPD en pacientes con AR. En Turquía, pacientes con AIJ presentaron una frecuencia de PPD negativa significativamente superior a la población sana $(24 \% \text { vs } 6,6 \%)^{11}$. Datos similares fueron descriptos en Perú, donde observaron una frecuencia de anergia a PPD del $70 \%$ en pacientes con AR en comparación a $26 \%$ en la población general'.

Nuestro estudio tiene algunas limitantes: primero, los pacientes fueron sólo monitoreados con PPD y no dispusimos de quantiferon debido a su alto costo. Segundo, todos los pacientes con viraje fueron tratados con ISH, por lo cual no sabemos qué sucedería si los pacientes no hubieran sido tratados con la profilaxis recomendada. Por último, el diseño de nuestro estudio no permite definir si realmente es necesaria la realización de PPD posterior al inicio del tratamiento biológico y en consecuencia el tratamiento profiláctico en caso de viraje.

En conclusión, el viraje en pacientes con enfermedades inflamatorias crónicas en terapia biológica en nuestra población fue bajo y solo un paciente desarrolló tuberculosis activa. Deberían realizarse otros estudios para comprobar riesgo/beneficio del monitoreo posterior de la PPD durante el tratamiento con agentes biológicos y el consecuente uso de tratamiento profiláctico.

\section{Bibliografía}

1. Listing J, Strangfekd A, Kary S, Rau R, von Hinueber $\mathrm{U}$, Stoyanova-Scholz M, et al. Infections in patients with rheumatoid arthritis treated with biologic agents. Arthritis Rheum 2005; 52(11):3403-12.

2. Wallis RS, Broder MS, Wong JY, Hanson ME, Beenhouwer DO. Granulomatous infectious diseases associated with tumor necrosis factor antagonists. CID 2004;38:1261-5.

3. Kindler V, Sappino AP, Grau GE, Piguet PF, Vassalli P. The inducing role of tumor necrosis factor in the development of bactericidal granulomas during BCG infection. Cell 1989;56(5):731-40.

4. Gómez-Reino JJ, Carmona L, Descalzo MA, Biobadaser Group. Risk of Tuberculosis in patients treated with tumor necrosis factor antagonists due to incomplete prevention of reactivation of latent infection. Arthritis Rheum 2007;57(5):756-61.

5. 1980-2009- Instituto Nacional de Enfermedades Respiratorias E. Coni. PRO.TB. Doc.Téc. No 16/09.

6. Ahmed AR, Blose DA. Delayed-type hypersensitivity skin testing. A review. Arch Dermatol 1983;119(11):93445.

7. Ponce de León D, Acevedo-Vásquez E, Sánchez-Torres A, Cucho M, Alfaro J, Perich R, et al. Attenuated response to purified protein derivative in patients with rheumatoid arthritis: study in a population with a high prevalence of tuberculosis. Ann Rheum Dis 2005;64(9):1360-1.

8. Menzies D. Interpretation of repeated tuberculin tests boosting, conversion, and reversion. Am J Respir Crit Care Med 1999;159(1):15-21.

9. Abbate E, Ballester D, Barrera L, Brian MC, Echazarreta A, Gaitán C, et al. Consenso Argentino de Tuberculosis. Rev Arg Med Resp 2009;9:61-69. 
10. Wang L, Tunner M, Elwood R. A. Meta-analysys of the effect of bacilli Calmette Guerin vaccination on tuberculin test measurements. Torax 2002; 57: 804-9.

11. Kiray E, Kasapcopur O, Bas V, Kamburoglu-Goksel A, Midilli K, Arisoy N, et al. Purified Protein Derivative Response in Juvenile Idiopathic Arthritis. J Rheumatol 2009;36(9):2029-32.

12. Arnett FC, Edworthy SM, Bloch DA,McShane DJ, Fries JF, Cooper et al. The American Rheumatism Association 1987 revised criteria for the classification of rheumatoid arthritis. Arthritis Rheum 1988;31(3):315-24.

13. Funovits J, Aletaha D, Bykerk V, Combe B, Dougados M, Emery P, et al. The 2010 American College of Rheumatology/European League Against Rheumatism classification criteria for rheumatoid arthritis: methodological report phase I. Ann Rheum Dis 2010;69(9):1589-95.

14. Petty RE, Southwood TR, Manners P, Baum J, Glass D, Goldenberg J, et al, International League of Associations for Rheumatology Classification of Juvenile Idiopathic Arthritis: Second Revision, Edmonton, 2001,The Journal of Rheumatology 2004;31(2):390-2.

15. Rudwaleit M, van der Heijde D, Landewé R, Listing J, Akkoc N, Brandt J, et al. The development of Assessment of SpondyloArthritis international Society classification criteria for axial spondyloarthritis (part II): validation and final selection. Ann Rheum Dis 2009;68(6):777-83.

16. Rudwaleit $M$, van der Heijde D, Landewé R, Akkoc N, Brandt J, Chou C, et al. The Assessment of SpondyloArthritis international Society classification criteria for peripheral spondyloarthritis and for spondyloarthritis in general. Ann Rheum Dis 2011;70(1):25-31

17. Taylor W, Gladman D, Helliwell P, Marchesoni A, Mease P, Mielants $\mathrm{H}$, and the CASPAR study group. Classification criteria for psoriatic arthritis. Development of new criteria from a large international study. Arthritis Rheum. 2006;54(8)2665-73.

18. Anderson P, Gual A, Colon J. Alcohol y atención primaria de la salud: informaciones clínicas básicas para la identificación y el manejo de riesgos y problemas. Washington, D.C.: OPS, (C) 2008.

19. Informe costo de canasta básica Septiembre del 2013, INDEC (Instituto Nacional De Estadísticas y Censos).

20. Maldonado Ficco H, Pérez Alamino R, Schneeberger EE, Maldonado Cocco JA, Citera G. Validación del cuestionario RAPID3 en una cohorte de pacientes con artritis reumatoidea temprana y establecida, y su correlación con otros índices de actividad. Rev Argent Reumatol 2011;22 (2):31-39.

21. Citera G, Maldonado Cocco JA, Moroldo M, Burgos-Vargas R, Anaya J, López I, et al. Validación de la versión en español de los cuestionarios de capacidad funcional BAS-
FI y actividad de la enfermedad BASDAI en pacientes con Espondilitis Anquilosante en cuatro países latinoamericanos. Rev Arg Reumatol 1999;10(Supl 1):25.

22. Citera G, Arriola MS, Maldonado Cocco JA, Rosemffet MG, Sánchez MM, Goñi MA, et al. Validation and cross cultural adaptation of an argentine spanish version of the health assessment questionnaire disability index. J Clin Rheum 2004;10(3):110-5.

23. Dixon WG, Watson K, Lunt M, Hyrich L, Silman AJ, British Society for Rheumatology Biologics Register, Rates of Serious Infection, Including Site-Specific and Bacterial Intracellular Infection, in Rheumatoid Arthritis Patients Receiving Anti-Tumor Necrosis Factor Therapy. Arthritis Rheum 2006;54(8):2368-76.

24. Park JH, Seo GY, Lee JS, Kim TH, Yoo DH. Positive Conversion of Tuberculin Skin Test and Performance if Interferon Realese Assay to Detect Hidden Tuberculosis Infection During Anti-Tumor Necrosis Factor Agent Trial. J Rheumatol 2009;36(10):2158-63. 